\title{
Vacuum Polarization of a Charged Massless Fermionic Field by a Magnetic Flux in the Cosmic String Spacetime
}

\author{
J. Spinelly ${ }^{1,2}$ *and E. R. Bezerra de Mello ${ }^{2} \dagger$ \\ 1.Departamento de Física-CCT \\ Universidade Estadual da Paraíba \\ Juvêncio Arruda S/N, C. Grande, PB \\ 2.Departamento de Física-CCEN \\ Universidade Federal da Paraíba \\ 58.059-970, J. Pessoa, PB \\ C. Postal 5.008 \\ Brazil
}

November 15, 2018

\begin{abstract}
We calculate the vacuum averages of the energy-momentum tensor associated with a massless left-handed spinor fields due to magnetic fluxes on idealized cosmic string spacetime. In this analysis three distinct configurations of magnetic fields are considered: i) a homogeneous field inside the tube, ii) a magnetic field proportional to $1 / r$ and iii) a cylindrical shell with $\delta$-function. In these three cases the axis of the infinitely long tubes of radius $R$ coincides with the cosmic string. In order to proceed with these calculations we explicitly obtain the Euclidean Feynman propagators associated with these physical systems.
\end{abstract}

*E-mail: spinelly@fisica.ufpb.br

†E-mail: emello@fisica.ufpb.br 
As we shall see, these propagators possess two distinct parts. The first are the standard ones, i.e., corresponding to the spinor Green functions associated with the massless fermionic fields on the idealized cosmic string spacetime with a magnetic flux running through the line singularity. The second parts are new, they are due to the finite thickness of the radius of the tubes. As we shall see these extra parts provide relevant contributions to the vacuum averages of the energy-momentum tensor.

\section{Introduction}

Different types of topological defects may have been formed during the phase transition in the early universe [1]. Depending on the topology of the vacuum manifold they are domain walls, strings, monopoles and textures. Cosmic strings have gained some interest recently since they are considered as a good candidate to explain some components of anisotropy on the cosmic microwaves background [2], gamma ray bursts [3], gravitational waves [4] and highest energy cosmic rays [5]. Also they are thought to be important for the structure formation in the universe due to their huge energy density per unit length [6].

A classical field theory model which presents stringlike solutions is the Abelian Higgs model [7]. These solutions, also called "vortices", correspond to infinitely long objects. They have a core radius proportional to the inverse of the Higgs mass and magnetic flux tube with radius proportional to the inverse of the gauge boson mass.

Coupling the Abelian Higgs model with gravity, Garfinkle [8] showed that, as in flat spacetime, there exist static cylindrically symmetric solutions representing vortices. He also shown that asymptotically the spacetime around the cosmic string is a Minkowiski one minus a wedge. Its core has a non-zero thickness, and the magnetic field vanishes outside of it. A complete analysis about the structure of the Higgs and magnetic fields near the $U(1)$-gauge cosmic strings can only be provided numerically.

The vacuum polarization effects due to a magnetic field confined in a tube

\footnotetext{
${ }^{1}$ For cosmic strings formed at grand unified theory, the energy densities is of order than $10^{21} \mathrm{Kg} / \mathrm{m}$ and their radius $10^{-32} \mathrm{~m}$.
} 
of finite radius in Minkowiski spacetime were first analysed by Serebryanyi [9]. A few years later, Guimarães and Linet [10] and Linet [11], calculated these effects for a charged massless scalar and fermionic fields, respectively, on a idealized cosmic string spacetime. There a magnetic field running through the line singularity was considered. As a consequence, the renormalized vacuum expectation value associated with the energy-momentum tensor, $\left\langle T_{\mu \nu}(x)\right\rangle_{\text {Ren. }}$, presents contributions coming from the geometry of the spacetime and also the magnetic flux. In order to develop these calculations the respective Green functions were obtained. More recently Sriramkumar [12] has calculated the vacuum fluctuations of current and energy densities for a massless charged scalar field around an idealized cosmic string carrying a magnetic flux. There the Green function was obtained taking into account the presence of the vector potential in the differential operator, $D_{\mu}=\partial_{\mu}-i e A_{\mu}$, which presents the advantage of calculating the two-point function without imposing any boundary condition on the field ${ }^{2}$.

The analysis of the vacuum polarization effects on a massless charged scalar field by a magnetic flux confined in a cylindrical tube of finite radius in a cosmic string background was performed in [13]. There three distinct configurations of magnetic field specified below have been considered. In all of them the axis of the infinitely long tube of radius $R$ coincides with the cosmic string. Calculating the renormalized vacuum expectation value (VEV) of the square of the field, $\left\langle\Phi^{*}(x) \Phi(x)\right\rangle_{\text {Ren }}$, and the energy-momentum tensor, $\left\langle T_{\mu}^{\nu}(x)\right\rangle_{\text {Ren }}$, it was observed that these quantities present two contributions for each model of magnetic flux. The first are the standard ones due to the conical geometry of the spacetime and the magnetic flux. The second contributions are corrections due to the finite thickness of the radius of the tube. These extra terms provided relevant contributions for points outside the tube. Specifically, for the third model this contribution is a long-range effect, i.e., it is as relevant as the standard one up to a distance which exceed the radius of the observable universe.

In this paper we return to this problem and extend the above analysis to the charged massless fermionic fields. As we shall see, the renormalized VEV of the energy-momentum tensors also present two distinct contribu-

\footnotetext{
${ }^{2}$ Absorbing the gauge field on the definition of the matter fields implies an overall phase shift in the Green function. However this procedure provides to $\left\langle T_{\mu \nu}(x)\right\rangle_{R e n}$. the same result as obtained considering explicitly the presence of the gauge field in the differential operator.
} 
tions. Moreover due mainly to interaction of the spin degrees of freedom of the fermionic fields with the magnetic fluxes, the corrections due to the nonzero thickness of the radius of the tube are composed by two terms. Unfortunately it is not possible to provide analytical informations about the radial behavior of these corrections, and only numerical analysis can do that. Also, additional couplings between the spin with the geometry are also present in this system.

The idealized model for a infinitely long straight static cosmic string spacetime can be given in cylindrical coordinates by the line element:

$$
d s^{2}=-d t^{2}+d r^{2}+\alpha^{2} r^{2} d \theta^{2}+d z^{2},
$$

where $\alpha=1-4 \mu$ is a parameter smaller than unity which codifies the presence of a conical two-surface $(r, \theta)$. In fact for a typical Grand Unified Theory, $\alpha=1-O\left(10^{-6}\right)$.

We shall consider the presence of a magnetic field along the $z$-direction assuming that the field has a finite range in the radial coordinate. We are particularly interested in the three models:

i) $H(r)=\frac{\phi}{\alpha \pi R^{2}} \Theta(R-r)$, homogeneous field inside;

ii) $H(r)=\frac{\phi}{2 \pi \alpha R r} \Theta(R-r)$, field proportional to $1 / r$;

iii) $H(r)=-\frac{\phi}{2 \pi \alpha R} \delta(r-R)$, cylindrical shell,

where $R$ is the radial extent of the tube, and $\phi$ is the total flux. The ratio of the flux to the quantum flux $\phi_{o}$, can be expressed by $\phi / \phi_{0}=N+\gamma$, where $N$ is the integer part and $0<\gamma<1$. $^{3}$

Although the structure of the magnetic field produced by a $U(1)$-gauge cosmic string cannot be presented by any analytical function, its influence on the vacuum polarization effects of charged matter fields takes place for sure. So in this case, the geometric and magnetic interactions provide contributions. The relevant physical question is how important they are. In this paper, together with previous ones, by Guimarães and Linet, Linet himself and us, we try to answer that question. In our analysis we added an extra ingredient on this investigation not yet considered.

As we have already mentioned, considering a finite thickness for the magnetic flux, additional contributions to the vacuum averages are obtained. Because there is no possibility to analysis these effects for a realistic gauge cosmic string, we consider idealized configurations to the magnetic field. This

\footnotetext{
${ }^{3}$ In this paper we are considering $\hbar=G=c=1$.
} 
allows us to develop an analytical procedure, and as we shall see, even for these configurations, some general informations regarding to the influence of the nonvanishing thickness of the magnetic flux on the vacuum polarization can be extracting.

This paper is organized as follows. In section 2, we explicitly construct the spinor Feynman propagators for three different models of magnetic fields. Having these Green functions, in section 3 we calculate the formal expression to the respective renormalized vacuum expectation values of the energymomentum tensor $\left\langle T_{\mu}^{\nu}(x)\right\rangle_{\text {Ren. }}$ associated with the left-handed fermionic fields. Because non standard contributions to these VEV do not provide concrete informations about their radial behavior, in section 4 we present our numerical analysis about them for specific values for the parameters $\alpha, \gamma$ and $N$. Finally we leave for section 5 our concluding remarks.

\section{Spinor Feynman Propagator}

The Feynman propagator associated with a charged fermionic field, $S_{F}\left(x, x^{\prime}\right)$, obeys the following differential equation:

$$
(i \not \nabla+e A-M) S_{F}\left(x, x^{\prime}\right)=\frac{1}{\sqrt{-g}} \delta^{4}\left(x, x^{\prime}\right) I_{4},
$$

where $g=\operatorname{det}\left(-g_{\mu \nu}\right)$. The covariant derivative operator reads

$$
\not=e_{(a)}^{\mu} \gamma^{(a)}\left(\partial_{\mu}+\Gamma_{\mu}\right)
$$

$e_{(a)}^{\mu}$ being the vierbein satisfying the condition $e_{(a)}^{\mu} e_{(b)}^{\nu} \eta^{(a)(b)}=g^{\mu \nu}$ and $\Gamma_{\mu}$ is the spin connection given in terms of flat spacetime $\gamma$ matrices by

$$
\Gamma_{\mu}=-\frac{1}{4} \gamma^{(a)} \gamma^{(b)} e_{(a)}^{\nu} e_{(b) \nu ; \mu},
$$

and

$$
A=e_{(a)}^{\mu} \gamma^{(a)} A_{\mu}
$$

In the Appendix we show that if a bispinor $D_{F}\left(x, x^{\prime}\right)$ satisfies the differential equation

$$
\begin{gathered}
{\left[-\mathcal{D}^{2}+\frac{1}{4} \mathcal{R}+\operatorname{ieg}^{\mu \nu}\left(D_{\mu} A \nu\right)-i e \Sigma^{\mu \nu} F_{\mu \nu}+2 i e g^{\mu \nu} A_{\nu} \nabla_{\nu}\right.} \\
\left.+e^{2} g^{\mu \nu} A_{\mu} A_{\nu}+M^{2}\right] D_{F}\left(x, x^{\prime}\right)=-\frac{1}{\sqrt{-g}} \delta^{4}\left(x, x^{\prime}\right) I_{4}
\end{gathered}
$$


with

$$
\Sigma^{\mu \nu}=\frac{1}{4}\left[\gamma^{\mu}, \gamma^{\nu}\right]
$$

$\mathcal{R}$ being the scalar curvature and the generalized d'Alembertian operator given by

$$
\mathcal{D}^{2}=g^{\mu \nu} \nabla_{\mu} \nabla_{\nu}=g^{\mu \nu}\left(\partial_{\mu} \nabla_{\nu}+\Gamma_{\mu} \nabla_{\nu}-\Gamma_{\mu \nu}^{\alpha} \partial_{\alpha}\right)
$$

then the spinor Feynman propagator may be written as

$$
S_{F}\left(x, x^{\prime}\right)=(i \not \nabla+e A+M) D_{F}\left(x, x^{\prime}\right) .
$$

Now after this brief introduction about the calculation of spinor Feynman propagator, let us specialize it to the cosmic string spacetime in the presence of a magnetic field along the $z$-direction. We shall choose the following base tetrad:

$$
e_{(a)}^{\mu}=\left(\begin{array}{cccc}
1 & 0 & 0 & 0 \\
0 & \cos \theta & -\sin \theta / \alpha r & 0 \\
0 & \sin \theta & \cos \theta / \alpha r & 0 \\
0 & 0 & 0 & 1
\end{array}\right) .
$$

In order to provide an explicit expression to the differential operators above, we shall adopt the same representation as in [15] to the $\gamma$-matrices in Minkowiski spacetime.

As to the four vector potential we have

$$
A_{\mu}=(0,0, A(r), 0)
$$

with

$$
A(r)=\frac{\phi}{2 \pi} a(r) .
$$

For the two first models, we can represent the radial function $a(r)$ by:

$$
a(r)=f(r) \Theta(R-r)+\Theta(r-R),
$$

with

$$
f(r)=\left\{\begin{array}{cc}
r^{2} / R^{2}, & \text { for the model }(i) \text { and } \\
r / R, & \text { for the model }(i i) .
\end{array}\right.
$$


For the third model,

$$
a(r)=\Theta(R-r) .
$$

In this spacetime the only non-zero spin connection is

$$
\Gamma_{2}=\frac{i}{2}(1-\alpha) \Sigma^{3}
$$

and for the Christofell symbols we have:

$$
\Gamma_{22}^{1}=-\alpha^{2} r, \quad \Gamma_{12}^{2}=\Gamma_{21}^{1}=1 / r
$$

Defining by $\mathcal{K}(x)$ the $4 \times 4$ matrix differential operator which acts on $D_{F}\left(x, x^{\prime}\right)$ in $(6)$, for this physical system we obtain:

$$
\begin{aligned}
\mathcal{K}(x) & =-\Delta-\frac{i}{\alpha^{2} r^{2}}(1-\alpha) \Sigma^{3} \partial_{\theta}-e H(r) \Sigma^{3}+\frac{1}{4 \alpha^{2} r^{2}}(1-\alpha)^{2} \\
& -\frac{e}{\alpha^{2} r^{2}}(1-\alpha) A(r) \Sigma^{3}+\frac{2 i e}{\alpha^{2} r^{2}} A(r) \partial_{\theta}+\frac{e^{2}}{\alpha^{2} r^{2}} A^{2}(r)+M^{2},
\end{aligned}
$$

where

$$
\Sigma^{3}=\left(\begin{array}{cc}
\sigma^{3} & 0 \\
0 & \sigma^{3}
\end{array}\right),
$$

and

$$
\Delta=-\partial_{t}^{2}+\partial_{r}^{2}+\frac{1}{r} \partial_{r}+\frac{1}{\alpha^{2} r^{2}} \partial_{\theta}^{2}+\partial_{z}^{2} .
$$

We can see that the differential operator above explicitly exhibits, besides the ordinary d'Alembertian operator on the conical spacetime, four different types of interaction terms: $(i)$ the usual charge-magnetic field, $(i i)$ the spinmagnetic field, (iii) the spin-geometry and (iv) the spin-charge-geometry. All of the last three interactions were absent in the analogous differential operator used to define the scalar Green function in [13].

Moreover, as we can see the operator $\mathcal{K}(x)$ is diagonal in $2 \times 2$ blocks. This means that the two upper components of the Dirac spinor interact with the gravitational and magnetic fields in similar way as the two lower components and they do not interact among themselves.

The system that we want to study consists of massless charged fermionic field in the cosmic string spacetime in the presence of an Abelian magnetic field along the $z$-direction. Let us chose a left-handed field. In this case the 
Dirac equation and the equation which defines the spinor Feynman propagator reduce themselves to a $2 \times 2$ matrix differential equation:

$$
\not D_{L} \chi=0
$$

where

$$
\not D_{L}=i\left[\partial_{t}-\sigma^{(r)}\left(\partial_{r}-\frac{\left(\alpha^{-1}-1\right)}{2 r}\right)-\frac{1}{\alpha r} \sigma^{(\theta)}\left(\partial_{\theta}-i A(r)\right)-\sigma^{(z)} \partial_{z}\right]
$$

with $\sigma^{(r)}=\vec{\sigma} \cdot \hat{r}, \sigma^{(\theta)}=\vec{\sigma} \cdot \hat{\theta}$ and $\sigma^{(z)}=\vec{\sigma} . \hat{z}$.

The Feynman two-components propagator obeys the equation

$$
i \not D_{L} S_{F}^{L}\left(x, x^{\prime}\right)=\frac{1}{\sqrt{-g}} \delta^{4}\left(x, x^{\prime}\right) I_{2}
$$

and can be given by

$$
S_{F}^{L}\left(x, x^{\prime}\right)=i \not D_{L} G^{L}\left(x, x^{\prime}\right)
$$

where now $G^{L}\left(x, x^{\prime}\right)$ obeys the $2 \times 2$ matrix differential equation:

$$
\bar{K}(x) G^{L}\left(x, x^{\prime}\right)=-\frac{1}{\sqrt{-g}} \delta^{4}\left(x, x^{\prime}\right) I_{2},
$$

with

$$
\begin{aligned}
\bar{K}(x) & =-\Delta-\frac{i}{\alpha^{2} r^{2}}(1-\alpha) \sigma^{3} \partial_{\theta}-e H(r) \sigma^{3}+\frac{1}{4 \alpha^{2} r^{2}}(1-\alpha)^{2} \\
& -\frac{e}{\alpha^{2} r^{2}}(1-\alpha) A(r) \sigma^{3}+\frac{2 i e}{\alpha^{2} r^{2}} A(r) \partial_{\theta}+\frac{e^{2}}{\alpha^{2} r^{2}} A^{2}(r) .
\end{aligned}
$$

Because of the peculiar diagonal form of the above operator, let us take for $G^{L}$ the following expression:

$$
G^{L}\left(x, x^{\prime}\right)=\left(\begin{array}{cc}
G_{+}\left(x, x^{\prime}\right) & 0 \\
0 & G_{-}\left(x, x^{\prime}\right)
\end{array}\right) .
$$

So the differential equation (24) reduces itself to the two independent ones:

$$
\begin{gathered}
{\left[\Delta \pm \frac{i}{\alpha^{2} r^{2}}(1-\alpha) \partial_{\theta}-\frac{(1-\alpha)^{2}}{4 \alpha^{2} r^{2}} \pm e H(r)-\frac{2 i e}{\alpha^{2} r^{2}} A(r) \partial_{\theta}\right.} \\
\left. \pm \frac{e}{\alpha^{2} r^{2}}(1-\alpha) A(r)-\frac{e^{2}}{\alpha^{2} r^{2}} A^{2}\right] G_{ \pm}\left(x, x^{\prime}\right)=\frac{1}{\sqrt{-g}} \delta^{4}\left(x, x^{\prime}\right) .
\end{gathered}
$$


Due to the cylindrical symmetry of this system each component of the Euclidean Green function can be expressed by

$$
G_{ \pm}\left(x, x^{\prime}\right)=\frac{1}{(2 \pi)^{3}} \sum_{n=-\infty}^{\infty} e^{i n\left(\theta-\theta^{\prime}\right)} \int_{-\infty}^{\infty} d k \int_{-\infty}^{\infty} d \omega e^{i k\left(z-z^{\prime}\right)} e^{i \omega\left(\tau-\tau^{\prime}\right)} g_{n}^{ \pm}\left(r, r^{\prime}\right)
$$

Before to specialize on the specific model, let us write down the nonhomogeneous differential equation obeyed by the unknown function $g_{n}^{ \pm}\left(r, r^{\prime}\right)$. Substituting (28) into (27) and using the standard representation to the delta function in the temporal, angular and $z$-coordinates, we arrive at the following differential equation for the unknown function $g_{n}^{ \pm}\left(r, r^{\prime}\right)$ :

$$
\begin{array}{r}
{\left[\frac{d^{2}}{d r^{2}}+\frac{1}{r} \frac{d}{d r}-\frac{1}{\alpha^{2} r^{2}}\left[n^{2} \pm n(1-\alpha \mp 2 n e A)+\frac{\left(1-\alpha^{2}\right)}{4} \mp e(1-\alpha) A(r)\right.\right.} \\
\left.\left.+e^{2} A^{2}\right]-\beta^{2} \pm H(r)\right] g_{n}^{ \pm}\left(r, r^{\prime}\right)=\frac{1}{\alpha r} \delta\left(r-r^{\prime}\right),
\end{array}
$$

where $\beta^{2}=k^{2}+\omega^{2}$.

It is of our main interest to investigate the vacuum polarization effect for external points to the magnetic flux. So, we shall consider solutions of (29) with both $r$ and $r^{\prime}$ greater than $R$.

Let us define by $g_{n}^{<}\left(r, r^{\prime}\right)$ the solution of (29) regular at $r \rightarrow 0$, and by $g_{n}^{>}\left(r, r^{\prime}\right)$ the solution that vanishes at infinity. These two solutions must satisfy continuity condition at $r=r^{\prime}$, with their first derivative discontinuous at this point. Integrating out in the region $r<R$ the inner solutions, corresponding to $r<r^{\prime}$, for the first two models we have:

$$
g_{n}^{ \pm<}\left(r, r^{\prime}\right)=A_{(i)}^{ \pm} H_{i}^{ \pm}(r)
$$

for $r<R$ and

$$
g_{n}^{ \pm<}\left(r, r^{\prime}\right)=B_{(i)}^{ \pm}\left[I_{\left|\nu_{ \pm}\right|}(\beta r)+E_{(i)}^{ \pm}(\beta R) K_{\left|\nu_{ \pm}\right|}(\beta r)\right]
$$

for $R<r<r^{\prime}$, where

$$
\nu_{ \pm}=\frac{\left(n \pm \frac{1-\alpha}{2}-\delta\right)}{\alpha}
$$


with $\delta=\frac{e \phi}{2 \pi}=N+\gamma \cdot H_{i}^{ \pm}(r)$, for $i=1,2$, represents the solution associated with the two first models:

$$
H_{1}^{ \pm}(r)=\frac{1}{r} M_{\sigma_{1( \pm)},\left|\lambda_{1( \pm)}\right|}\left(\frac{\delta}{\alpha R^{2}} r^{2}\right),
$$

and

$$
H_{2}^{ \pm}(r)=\frac{1}{\sqrt{r}} M_{\sigma_{2( \pm)},\left|\lambda_{2( \pm)}\right|}(\zeta r)
$$

with

$$
\begin{gathered}
\sigma_{1( \pm)}=\frac{n}{2 \alpha}-\frac{\beta^{2} R^{2} \alpha}{4 \delta} \pm \frac{\alpha+1}{4 \alpha} \\
\lambda_{1( \pm)}=\frac{n}{2 \alpha} \pm \frac{1-\alpha}{4 \alpha} \\
\sigma_{2( \pm)}=\frac{\delta(2 n \pm 1)}{2 \alpha\left(\delta^{2}+\beta^{2} \alpha^{2} R^{2}\right)^{1 / 2}}
\end{gathered}
$$

and

$$
\lambda_{2( \pm)}=\frac{n}{\alpha} \pm \frac{1-\alpha}{2 \alpha} \text {. }
$$

For both functions $H_{2}^{ \pm}, \zeta=\frac{2}{R \alpha}\left(\delta^{2}+\beta^{2} R^{2} \alpha^{2}\right)^{1 / 2}$.

In both cases $M_{\sigma, \lambda}$ represents the Whittaker function. The constant $E_{(i)}^{ \pm}(\beta R)$ is determined by matching $g_{n}^{ \pm<}$and its first derivative at $r=R$. So we get

$$
E_{(i)}^{ \pm}(\beta R)=\frac{H_{i}^{\prime \pm}(R) I_{\left|\nu_{ \pm}\right|}(\beta R)-H_{i}^{ \pm}(R) I_{\left|\nu_{ \pm}\right|}^{\prime}(\beta R)}{H_{i}^{ \pm}(R) K_{\left|\nu_{ \pm}\right|}^{\prime}(\beta R)-H_{i}^{\prime \pm}(R) K_{\left|\nu_{ \pm}\right|}(\beta R)} .
$$

In all the expressions, $I_{\nu}$ and $K_{\nu}$ are the modified Bessel functions.

The outer solution of (29) is given by

$$
g_{n(i)}^{ \pm>}\left(r, r^{\prime}\right)=D_{(i)}^{ \pm} K_{\left|\nu_{ \pm}\right|}(\beta r), \quad \text { for } r>r^{\prime} .
$$

Now, imposing the boundary conditions on $g_{n}^{ \pm<}$and $g_{n}^{ \pm>}$at $r=r^{\prime}$, we get the following result:

$$
g_{n}^{ \pm}\left(r, r^{\prime}\right)=-\frac{1}{\alpha}\left[I_{|\nu \pm|}\left(\beta r^{<}\right)+E_{(i)}^{ \pm}(\beta R) K_{|\nu \pm|}\left(\beta r^{<}\right)\right] K_{|\nu \pm|}\left(\beta r^{>}\right) .
$$

In the above equation $r^{>}\left(r^{<}\right)$is the larger (smaller) value between $r$ and $r^{\prime}$. Substituting (41) into (28), developing the sum in $n$ and integrating over $k$ 
and $\omega$ for the first part, the Euclidean Green functions acquire the following expression:

$$
\begin{aligned}
G_{ \pm}\left(x, x^{\prime}\right)= & -\frac{e^{i N \Delta \theta}}{8 \pi^{2} \alpha r r^{\prime} \sinh u_{0}} \frac{e^{\mp i \Delta \theta} \sinh \left(\gamma^{ \pm} u_{0} / \alpha\right)+\sinh \left[\left(1-\gamma^{ \pm}\right) u_{0} / \alpha\right]}{\cosh \left(u_{0} / \alpha\right)-\cos \Delta \theta} \\
- & \frac{1}{4 \pi^{2} \alpha} \int_{0}^{\infty} d \beta \beta J_{0}\left(\beta \sqrt{(\Delta \tau)^{2}+(\Delta z)^{2}}\right) \\
& \sum_{n} e^{i n \Delta \theta} E_{(i)}^{ \pm}(\beta R) K_{\left|\nu_{ \pm}\right|}(\beta r) K_{\left|\nu_{ \pm}\right|}\left(\beta r^{\prime}\right),
\end{aligned}
$$

with $\gamma^{ \pm}=(1-\alpha) / 2 \mp \gamma$ and

$$
\cosh u_{o}=\frac{r^{2}+r^{\prime 2}+(\Delta \tau)^{2}+(\Delta z)^{2}}{2 r r^{\prime}} .
$$

As we can observe, the first term in these Green functions depends only on the conicity parameter and the fractional part of $\phi / \phi_{0}$. Moreover, the second part contains information about the radius of the magnetic tube through the constants $E_{(i)}^{ \pm}$.

As to the third model, the solution to $g_{n}^{ \pm}\left(r, r^{\prime}\right)$ is:

$$
g_{n}^{ \pm<}\left(r, r^{\prime}\right)=A^{ \pm} I_{\left|\nu_{ \pm}\right|}(\beta r)
$$

for $r<R$,

$$
g_{n}^{ \pm<}\left(r, r^{\prime}\right)=B^{ \pm}\left[I_{\left|\epsilon_{ \pm}\right|}(\beta r)+E^{ \pm}(\beta R) K_{\left|\epsilon_{ \pm}\right|}(\beta r)\right]
$$

for $R<r<r^{\prime}$ and

$$
g_{n}^{ \pm>}\left(r, r^{\prime}\right)=D^{ \pm} K_{\left|\epsilon_{ \pm}\right|}(\beta r),
$$

for $r>r^{\prime}$. In the above equation $\epsilon_{ \pm}=1 / \alpha(n \pm(1-\alpha) / 2)$. Again, the coefficients $A^{ \pm}, B^{ \pm}$and $D^{ \pm}$can be determined by imposing boundary conditions at $r=R$ and $r=r^{\prime}$. However, due to the expression of the magnetic field in this model is concentrate as a $\delta$-function at the cylindrical shell, there happens a discontinuity condition in the first derivative of $g^{ \pm<}$at $r=R$. The rest being the same. So using these facts we obtain:

$$
g_{n}^{ \pm}\left(r, r^{\prime}\right)=-\frac{1}{\alpha}\left[I_{\left|\epsilon_{ \pm}\right|}\left(\beta r^{<}\right)+E^{ \pm}(\beta R) K_{\left|\epsilon_{ \pm}\right|}\left(\beta r^{<}\right)\right] K_{\left|\epsilon_{ \pm}\right|}\left(\beta r^{>}\right)
$$


where now

$$
E^{ \pm}(\beta R)=\frac{S^{ \pm}(\beta R)}{P^{ \pm}(\beta R)}
$$

with

$S^{ \pm}(\beta R)=I_{\left|\epsilon_{ \pm}\right|}(\beta R) I_{\left|\nu_{ \pm}\right|}^{\prime}(\beta R)-I_{\left|\nu_{ \pm}\right|}(\beta R) I_{\left|\epsilon_{ \pm}\right| / \alpha}^{\prime}(\beta R) \pm \frac{\delta}{\alpha R} I_{\left|\epsilon_{ \pm}\right|}(\beta R) I_{\left|\nu_{ \pm}\right|}(\beta R)$

and

$P^{ \pm}(\beta R)=I_{\left|\nu_{ \pm}\right|}(\beta R) K_{\epsilon_{ \pm}}^{\prime}(\beta R)-I_{\left|\nu_{ \pm}\right|}^{\prime}(\beta R) K_{\left|\epsilon_{ \pm}\right|}(\beta R) \mp \frac{\delta}{\alpha R} K_{\left|\epsilon_{ \pm}\right|}(\beta R) I_{\left|\nu_{ \pm}\right|}(\beta R)$.

Finally, substituting the expression found to $g_{n}^{ \pm}$above into (28), and adopting similar procedure as we did in the two previous cases, we obtain:

$$
\begin{aligned}
G_{ \pm}\left(x, x^{\prime}\right)= & -\frac{1}{8 \pi^{2} \alpha r r^{\prime} \sinh u_{0}} \frac{e^{i \mp \Delta \theta} \sinh \left(\bar{\gamma} u_{0} / \alpha\right)+\sinh \left[(1-\bar{\gamma}) u_{0} / \alpha\right]}{\cosh \left(u_{o} / \alpha\right)-\cos \Delta \theta} \\
- & \frac{1}{4 \pi^{2} \alpha} \int_{0}^{\infty} d \beta \beta J_{0}\left(\beta \sqrt{(\Delta \tau)^{2}+(\Delta z)^{2}}\right) \times \\
& \sum_{n=-\infty}^{\infty} e^{i n \Delta \theta} E^{ \pm}(\beta R) K_{\mid \epsilon \pm}(\beta r) K_{\mid \epsilon \pm}\left(\beta r^{\prime}\right),
\end{aligned}
$$

with $\bar{\gamma}=(1-\alpha) / 2$.

Here the first term of the Green function depends only on the conicity parameter.

Although all expressions to $g_{n}^{<}$and $g_{n}^{>}$present dependence on the radial coordinates $r$ and $r^{\prime}$, their dependence on $r^{\prime}$ are implicitly contained in the coefficients which multiply the functions of $r$. These dependences appear as consequence of the boundary conditions obeyed by them at $r=r^{\prime}$.

\section{Computation of $\left\langle\hat{T}_{00}\right\rangle_{\text {Ren }}$}

The vacuum expectation value of the energy-momentum tensor associated with the system under investigation can be obtained in the book by Grib et al [14], combining respectively the classical expressions to this tensor associated with the fermionic field in presence of electromagnetic interaction in a curved spacetime. It is given by:

$$
\left\langle\hat{T}_{\mu \nu}\right\rangle=\frac{1}{4} \lim _{x^{\prime} \rightarrow x} \operatorname{tr}\left[\sigma_{\mu}\left(D_{\nu}-\bar{D}_{\nu^{\prime}}\right)-\sigma_{\nu}\left(D_{\mu}-\bar{D}_{\mu^{\prime}}\right)\right] S_{F}^{L}\left(x, x^{\prime}\right),
$$


where $D_{\sigma}=\nabla_{\sigma}-i e A_{\sigma}$, the bar denotes complex conjugate and $\sigma^{\mu}=$ $\left(I_{2}, \sigma^{(r)}, \sigma^{(\theta)}, \sigma^{(z)}\right)$.

In order to take into account the presence of the three magnetic field configurations, given previously, we write the vector potential in the form $A_{\mu}=\left(0,0, \frac{\phi a(r)}{2 \pi}, 0\right)$, with $a(r)$ being given by (12) and (13), for the first two cases and by (14) for the third case. The spinor Green functions are expressed in terms of the bispinor $G^{L}\left(x, x^{\prime}\right)$ given by $(26)$ with $G_{ \pm}\left(x, x^{\prime}\right)$ given by (42) for the two first models and (49) for the third one.

For simplicity let us calculate $\left\langle T_{00}(x)\right\rangle$ only. The other components of this tensor can be obtained by using the conservation condition

$$
\nabla_{\mu}\left\langle T_{\nu}^{\mu}\right\rangle_{\text {Ren. }}=0
$$

and the vanishing of trace ${ }^{4}$

$$
\left\langle T_{\mu}^{\mu}\right\rangle_{\text {Ren } .}=0
$$

Fortunately only the second order time derivative provides a nonzero contribution to $\left\langle T_{00}(x)\right\rangle$. In fact all the other terms go to zero in the coincidence limit and/or after taking the trace over the Pauli matrices. Moreover, because the bispinor depends on the time variable with $t-t^{\prime}$, we finally have:

$$
\begin{aligned}
\left\langle T_{00}(x)\right\rangle & =-\lim _{x^{\prime} \rightarrow x} \operatorname{tr}\left(\partial_{t}^{2} G\left(x, x^{\prime}\right)\right) \\
& =\lim _{x^{\prime} \rightarrow x} \operatorname{tr}\left(\partial_{\tau}^{2} G_{E}\left(x, x^{\prime}\right)\right),
\end{aligned}
$$

where we have made a Wick rotation on the above equation.

However the calculation of the above expression provides a divergent result. In order to obtain a finite and well defined expression, we must apply in this calculation some renormalization procedure. The method which we shall apply here is the point-splitting renormalization procedure. The basic idea of this method consists of subtracting from the Green function all the divergences which appear in the coincidence limit. In [16] Adler et al observed that the singular behavior of the Green function in the coincidence limit has the same structure as the Hadamard one. Later Wald [17] introduced a

\footnotetext{
${ }^{4}$ In general the trace of the renormalized VEV of the energy-momentum tensor is equal to $\frac{1}{16 \pi^{2}} \operatorname{Tra}_{2}[18]$. However, because this spacetime is locally flat and there is no magnetic field outside the tube, $a_{2}=0$.
} 
modification to this technique in order to provide the correct result for the trace anomaly. In this way the renormalized vacuum expectation value of the zero-zero component of the energy-momentum tensor can be given by:

$$
\left\langle T_{00}(x)\right\rangle_{\text {Ren } .}=\lim _{x^{\prime} \rightarrow x} \operatorname{tr}\left[\partial_{\tau}^{2} G_{E}\left(x, x^{\prime}\right)-\partial_{\tau}^{2} G_{H}\left(x, x^{\prime}\right)\right] .
$$

Because this spacetime is locally flat, the Hadamard function coincides with the Euclidean Green function in a flat spacetime:

$$
G_{H}\left(x, x^{\prime}\right)=\frac{1}{4 \pi} \frac{1}{\left(x-x^{\prime}\right)^{2}} I_{2}
$$

As it was already mentioned, (42) and (49) present two distinct contributions; the first ones contain informations about the geometrical structure of the spacetime and the fractional part of the magnetic flux, and the second, the corrections due to the nonzero thickness of the radius of tube. Moreover, in the calculation of the $\mathrm{VEV}$, only their first contributions are divergent in the coincidence limit, the second ones are finite. Finally, explicitly exhibiting these remarks, we write down the renormalized VEV of the zero-zero component of the energy-momentum tensor by:

$$
\left\langle T_{00}(x)\right\rangle_{\text {Ren. }}=\left\langle T_{00}(x)\right\rangle_{\text {Reg. }}+\left\langle T_{00}(x)\right\rangle_{C},
$$

where

$$
\left\langle T_{00}(x)\right\rangle_{\text {Reg. }}=\lim _{x^{\prime} \rightarrow x}\left[\partial_{\tau}^{2} G_{+}\left(x, x^{\prime}\right)+\partial_{\tau}^{2} G_{-}\left(x, x^{\prime}\right)-2 \partial_{\tau}^{2} G_{H}\left(x, x^{\prime}\right)\right]
$$

and

$$
\left\langle T_{00}(x)\right\rangle_{C}=\lim _{x^{\prime} \rightarrow x} \partial_{\tau}^{2} G_{C}\left(x, x^{\prime}\right) .
$$

Here $G_{C}\left(x, x^{\prime}\right)$ represents the corrections due to the second terms in $G_{+}$and $G_{-}$for the three models, as shown next. After some intermediate calculations we arrive to the following results:

i) For the two first models,

$$
\begin{aligned}
\left\langle T_{00}(x)\right\rangle_{\text {Ren. }}= & \frac{1}{5760 \pi \alpha^{4} r^{4}}\left[\left(\alpha^{2}-1\right)\left(17 \alpha^{2}+7\right)+120 \gamma^{2}\left(\alpha^{2}-2 \gamma^{2}-1\right)\right] \\
& +\frac{1}{4 \pi^{2} r^{4} \alpha} \int_{0}^{\infty} d v v^{3} \sum_{n=-\infty}^{\infty}\left[E_{(i)}^{+}(v R / r) K_{\left|\nu_{+}\right|}^{2}(v)\right. \\
& \left.+E_{(i)}^{-}(v R / r) K_{\left|\nu_{-}\right|}^{2}(v)\right]
\end{aligned}
$$


for $i=1$ and 2 .

ii) For the third model,

$$
\begin{aligned}
\left\langle T_{00}(x)\right\rangle_{\text {Ren. }}= & \frac{1}{5760 \pi \alpha^{4} r^{4}}\left(\alpha^{2}-1\right)\left(17 \alpha^{2}+7\right) \\
& +\frac{1}{4 \pi^{2} r^{4} \alpha} \int_{0}^{\infty} d v v^{3} \sum_{n=-\infty}^{\infty}\left[E^{+}(v R / r) K_{|\epsilon+|}^{2}(v)\right. \\
& \left.+E^{-}(v R / r) K_{|\epsilon-|}^{2}(v)\right] .
\end{aligned}
$$

For all the above expressions the coefficients $E^{ \pm}$were given in (39) and (48).

The new terms obtained in (59) and (60) are consequence of the finite thickness of the magnetic flux and will be present even in the absence of cosmic string. Unfortunately it is not possible to evaluate these corrections analytically. The reason is because the dimensionless variable of integration $v$ appears not only in the argument of the modified Bessel function, but mainly because it appears in the order of the Whittaker functions through the factors $E^{ \pm}$.

Before to provide some qualitative information about the second contributions of (59) and (60), we would like to make a few comments about our results: $(i)$ The first contributions to them depend only on the conicity parameter $\alpha$, and the fractional part of $\phi / \phi_{0}$, denoted by $\gamma$, for the two first models. (ii) The second contributions, the corrections, vanish in the limit $R \rightarrow 0$. They also depend on the integer part of $\phi / \phi_{0}, N$.

Some qualitative informations about the behaviors of the second contributions can be provided: although for the three models these contributions present an overall $1 / r^{4}$ dependence on the radial coordinate, there exist an additional dependences in their integrands by the coefficients $E^{ \pm}$. However quantitative informations about these new behaviors can only be provided numerically. So we leave for the next section this analysis.

\section{Numerical Analysis of Corrections}

In this section we shall exhibit the quantitative behavior for corrections to the renormalized VEV of the zero-zero components of the energy-momentum tensor for the system analysed, considering for the three models specific values of the parameters $\alpha, \gamma$ and $N$. These behavior can only be provided by numerical analysis. 
The numerical method adopted by us to develop this analysis was numerical routine of MAPLE to evaluate integrals. Our first general conclusion is about the contributions presented by each term in the corrections. Although the integrals in the variable $v$ present contributions with opposite sign due to the coefficient $E^{+}$and $E^{-}$, they do not cancel each other. This fact is a consequence, mainly, to the preferential spatial direction dictated by the magnetic field on the spinor degrees of freedom.

Moreover, although the total contributions to the renormalized VEV of the zero-zero component of the energy-momentum tensor, $\left\langle T_{00}\right\rangle_{\text {Ren. }}$, requires in principal an infinite sum of terms, in fact this is not really necessary. The sums are very well represented by a few terms only. Expressing this quantity as

$$
\left\langle T_{00}\right\rangle_{\text {Ren. }}=\left\langle T_{00}\right\rangle_{\text {Reg. }}\left(1-\Delta_{\gamma, \alpha, N}(x)\right),
$$

where $x=R / r$, in our graphs we exhibit only the function $\Delta_{\gamma, \alpha, N}$ for the three models, considering $x$ running in the interval [0.01,0.1]. We also compare these functions for the three models. Although, we expect that this function goes to zero in the limit as $x \rightarrow 0$, or $r \rightarrow \infty$, our numerical results are not hundred percent trustful for $x$ smaller than $10^{-4}$. In fact the behavior of the factors $E_{(i)}^{ \pm}(v x)$ for the two first models and $E^{ \pm}(v x)$ for the third one for small value of $x$ can be obtained by developing a series expansion in power of their argument. Our results for them are:

$$
E_{(i)} \simeq(v x)^{2\left|\nu_{ \pm}\right|}
$$

with

$$
\nu_{ \pm}=\frac{1}{\alpha}\left[n \pm \frac{(1-\alpha)}{2}-\delta\right]
$$

and

$$
E^{ \pm} \simeq(v x)^{2\left|\epsilon_{ \pm}\right|}
$$

with

$$
\epsilon_{ \pm}=\frac{1}{\alpha}\left[n \pm \frac{(1-\alpha)}{2}\right]
$$

From these expressions we can infer that the corrections in fact go to zero for large values of the radial distance $r$, i.e., small value of $x$. However the numerical program provides very irregular diagrams to the integrands of (59) and (60) for small values of the dimensionless variable $v$ when $x$ becomes 
smaller than $10^{-4}$, so we can not trust on the evaluations of the integrals for these cases.

In Figs. 1(a) and 1(b), we present the radial dependence of the function $\Delta_{\gamma, \alpha, N}$, for $N=1$ and $N=2$, respectively, considering, $\alpha=0.99$ and $\gamma=0.02$. From both figures we can see that the shapes of the curves are very similar, however the larger contributions are due to the third models. By our numerical analysis these functions are bigger than unity, indicating that the corrections are in fact more relevant than the standard results, $\left\langle T_{00}\right\rangle_{\text {Reg. }}$, up to a distance one thousand times or, even more, larger than the radius of the tube. In the Figs. $2(a)$ and $2(b)$ we exhibit the logarithmic behavior for the same functions for a smaller scale of the variable $x$. In the latter is possible to infer a power behavior for the corrections as expected by analytical analysis.

In Fig. 3, we exhibit the functions $\Delta_{\gamma, \alpha, N}$ for $N=1$ and $N=2$. From this graph we observe that the correction due to $N=2$ is larger that for $N=1$. This result is expected, of course. However we know that the values adopted here to the magnetic fluxes are only few times larger than the quantum flux, so increasing this magnetic flux for sufficiently large value of $N$ the corrections becomes more and more relevant than the standard values of $\left\langle T_{00}\right\rangle_{\text {Reg. }}$.

Finally in Fig. 4, we exhibit the behavior of $\Delta_{\gamma, \alpha, N}$ varying the parameter $\alpha$ for $N=1$. From this graphs we observe a prominent increase for this factor for values of $\alpha$ bigger than 0.95. Moreover we can observe too that this effect becomes more evident in the third model of magnetic field. A similar graph has been constructed considering $N=2$, however no significant changes were found.

\section{Concluding Remarks}

In this paper we have explicitly exhibited the spinor Green functions associated with a left-handed charged spinor field on a cosmic string spacetime in the presence of external magnetic fluxes for three different configurations, all of them confined inside a long tube of radius $R$. Having these Green functions, we calculated the formal expressions to the renormalized VEV of the zero-zero component of the energy-momentum tensor, $\left\langle T_{00}(x)\right\rangle_{\text {Ren }}$. In these calculations we observe that two independent contributions were obtained. 
The first contributions are the standard ones due to the conical geometry of the spacetime and the fractional part of $\phi / \phi_{0}$. They coincide with the expression found by Linet in [11]. The second contributions are corrections due to the finite thickness of the radius of the tube. Unfortunately, it is not possible, by analytical analysis, to provide the complete information about the dependence of these parts with the radial coordinate, and only by numerical analysis we can do this. From this analysis we could observe that the corrections are more relevant than the standard results for distances up to $10^{3}$ bigger than the radius of the tube. Our results also suggest that the corrections are almost independent of the specific form of magnetic field, and that they increase for higher values of the magnitude of the magnetic field. Analysing the behavior of the corrections, $\Delta_{\gamma, \alpha, N}$ as function of $\alpha$, we also observe that they become more relevant when this parameter becomes closer than unity.

So, from all our numerical analysis we conclude that considering a nonvanishing radial extension to the magnetic flux in the calculation of the vacuum polarization, very important contributions take place. In fact these contributions become more relevant than the standard ones. The main reason for this fact is because no matter how big is the magnitude of the magnetic flux, the standard contribution depends only on the fractional part of $\phi / \phi_{0}$.

Although the models investigated here in fact do not correspond to the realistic configuration associated with a $U(1)$-gauge cosmic string, they provide some improvement in the calculation of vacuum polarization effects when compared with the ideal case; moreover our results give some general informations. They suggest that considering the influence of the nonzero thickness of the radial extent of the magnetic filed, relevant contributions to the induced energy densities associated with massless spinor fields are obtained, and more, these corrections are almost insensitive to the specific form of the magnetic fields.

Allen et all [20] have investigated the vacuum polarization of a massless scalar field on the cosmic string spacetime considering generically the effect of the internal structure of the string's core on the metric tensor. In this analysis the respective Green function also presents two parts, being one of them due to the non-zero core radius of the string. A more realistic treatment of the vacuum polarization effect associated with a charged field by a gauge cosmic string, must to consider the effect of both non-zero thicknesses for the magnetic field and the metric tensor. This analysis deserves to be analyzed 
appropriately in near future.

\section{Acknowledgments}

J. S. wants to thank the support given by the physics department of Universidade Estadual da Paraíba (UEPb) and E. R. B. M. to Conselho Nacional de Desenvolvimento Científico e Tecnológico (CNPq.) for partial financial support.

\section{Appendix. Square of the Dirac Operator}

In this appendix we want to find the square of the Dirac operator $i \not D=$ $-\not \nabla+i e A$. The result presents three distinct contributions.

a) The gauge field independent term ${ }^{5}$ :

$$
\gamma^{\mu} \nabla_{\mu} \gamma^{\nu} \nabla_{\nu}=-\mathcal{D}^{2}+\frac{1}{4} \mathcal{R}
$$

being $\mathcal{R}$ the scalar curvature of the manifold and $\mathcal{D}^{2}$ the generalized d'Alembertian operator.

b) The term linear in the gauge field has two contributions:

$$
-i e\left\{\gamma^{\mu} \nabla_{\mu} A_{\nu} \gamma^{\nu}+A_{\mu} \gamma^{\mu} \gamma^{\nu} \nabla_{\nu}\right\}
$$

which after some intermediate steps can be written as:

$$
i e\left\{g^{\mu \nu} D_{\mu} A_{\nu}-\Sigma^{\mu \nu} F_{\mu \nu}+2 g^{\mu \nu} A_{\nu} \nabla_{\mu}\right\},
$$

where

$$
\Sigma^{\mu \nu}=\frac{1}{4}\left[\gamma^{\mu}, \gamma^{\nu}\right] .
$$

The second term in (68) is a Pauli interaction one. It appears as a consequence of the square of the Dirac operator. $F_{\mu \nu}$ is the usual second-order, antisymmetric field-strength tensor.

c) As to the square gauge field term, it is easily obtained being equal to:

$$
e^{2} g^{\mu \nu} A_{\mu} A_{\nu}
$$

In our calculations above we have considered that the $\gamma$ Dirac matrices obey the anticommutator relation $\left\{\gamma^{\mu}, \gamma^{\nu}\right\}=-2 g^{\mu \nu}$.

\footnotetext{
${ }^{5}$ This part is very well known. It is given in the book by Birrell and Davies [19]
} 


\section{References}

[1] A. Vilenkin and E. P. S. Shellard, Cosmic Strings and Other Topological Defects (Cambridge University Press, Cambridge, 1994).

[2] S. Sarangi and S. -H. Henry Tye, Phys. Lett. B 536, 185 (2002), and K. Benabed and F. Bernardeu, Phys. Rev. D 62, 123510 (2000).

[3] V. Berezinski, B. Hnatyk and A. Vilenkin, Phys. Rev. D 64, 043004 (2001).

[4] T. Damour and A. Vilenkin, Phys. Rev. Lett. 85, 3761 (2000).

[5] P. Bhattacharjee and G. Sigl, Phys. Rep. 327, 109 (2000).

[6] T. W. B. Kibble and M. Hindmarsh, Rep. Prog. Phys. 58, 477 (1995).

[7] N. B. Nielsen and P.Olesen, Nucl. Phys. B61, 45 (1973).

[8] D. Garfinkle, Phys. Rev. D 32, 1323 (1985).

[9] E. M. Serebryanyi, Theor. Math. Phys. 64, 846 (1985).

[10] M. E. X. Guimarães and B. Linet, Commun. Math. Phys. 165, 297 (1994).

[11] B. Linet, J. Math. Phys. 36, 3694 (1995).

[12] L. Sriramkumar, Class. Quantum Grav. 18, 1015 (2001).

[13] J. Spinelly and E. R. Bezerra de Mello, Class. Quantum Grav. 20, 873 (2003); J. Spinelly and E. R. Bezerra de Mello, Int. J. Mod. Phys. A 17, 4375 (2002).

[14] A. A. Grib, S. G. Mamayev and V. M. Mostepanenko, Vacuum Quantum Effects in Strong Fields (Friedmann Laboratory Publishing, St. Petersburg, 1994).

[15] J. D. Bjorken and S. D. Drell, Relativistic Quantum Mechanics (McGraw-Hill, New York, 1964). 
[16] S. L. Adler, J. Lieberman and Y. J. Ng, Ann. Phys. (NY) 106, 279 (1977).

[17] Robert M. Wald, Phys. Rev. D 17, 1477 (1978).

[18] S. M. Christensen and M. J. Duff, Phys. Lett. 76B, 571 (1978).

[19] N. D. Birrell and P. C. W. Davies, Quantum Filed in Curved Space (Cambridge University Press. Cambridge, England, 1982).

[20] B. Allen, B. S. Kay and A. C. Ottewill, Phys. Rev. D 53, 6829 (1996).

\section{$7 \quad$ Figure Captions}

Figure 1: These graphs show the behavior of $\Delta_{\gamma, \alpha, N}$ for the three specific models of magnetic field configurations with: (a) $N=1, \alpha=0.99$ and $\gamma=0.02$. In $(b)$ we change to $N=2$.

Figure 2: These graphs present the logarithmic behavior of the previous one for $N=1$ in smaller scale of the variable $x$.

Figure 3: This graph shows the behavior of $\Delta_{\gamma, \alpha, N}$, for the first model in the case $N=1$ and $N=2$, considering $\alpha=0.99$ and $\gamma=0.02$.

Figure 4: This diagram presents the behavior of the correction $\Delta_{\gamma, \alpha, N}$ as function of $\alpha$. It was considered $x=10^{-1}, N=1$ and $\delta=0.002$. 


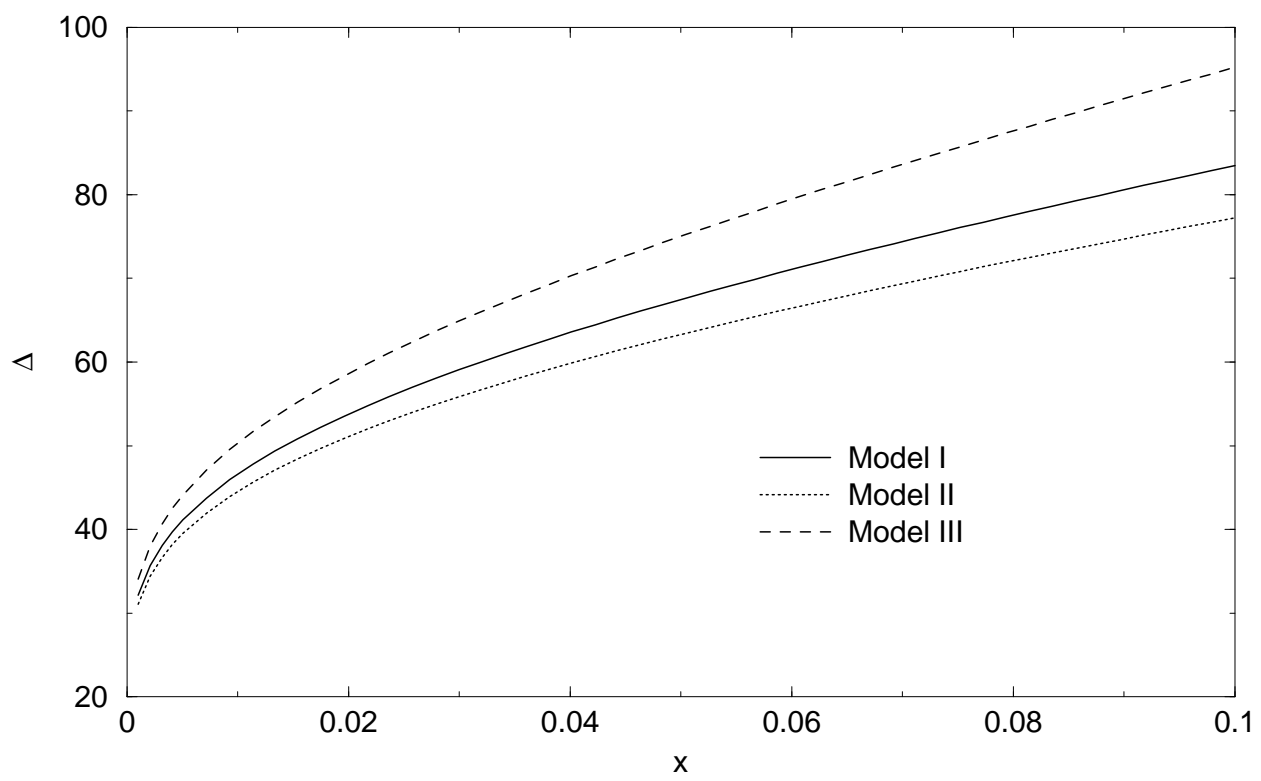

Fig. 1.a

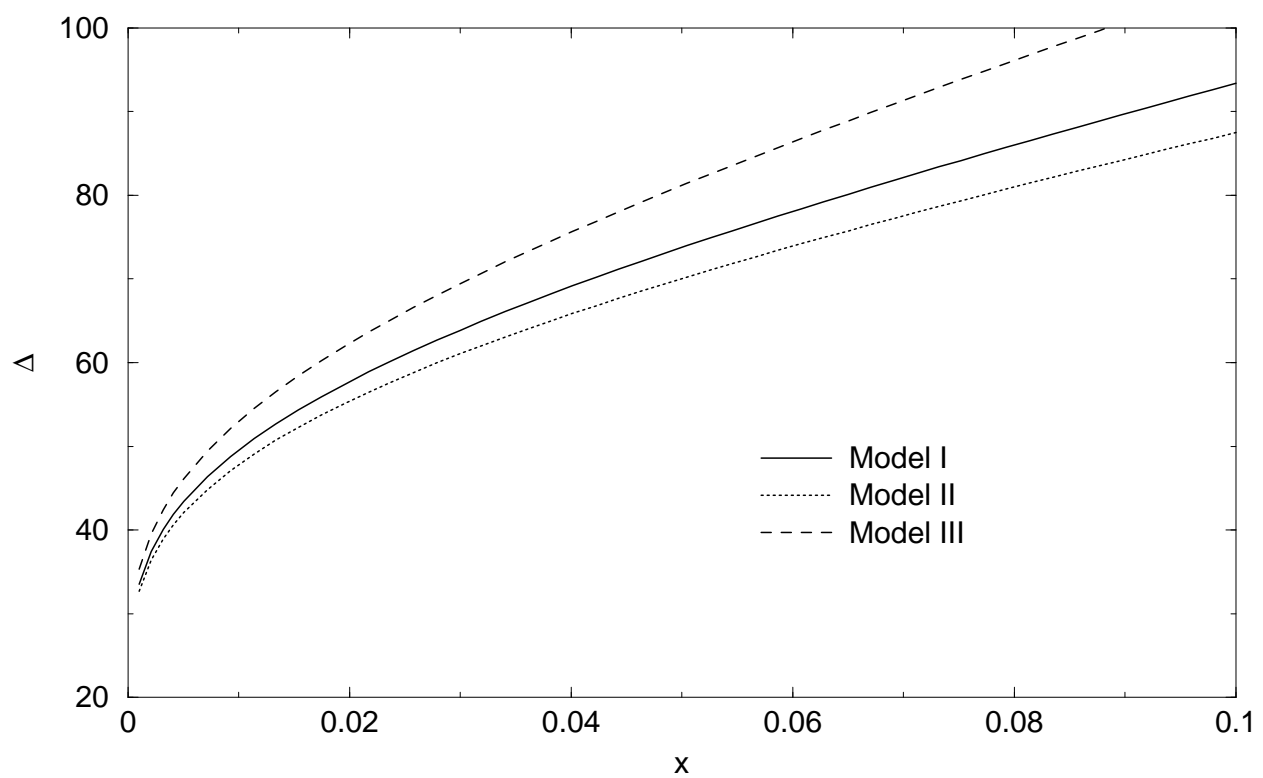

Fig. 1.b 


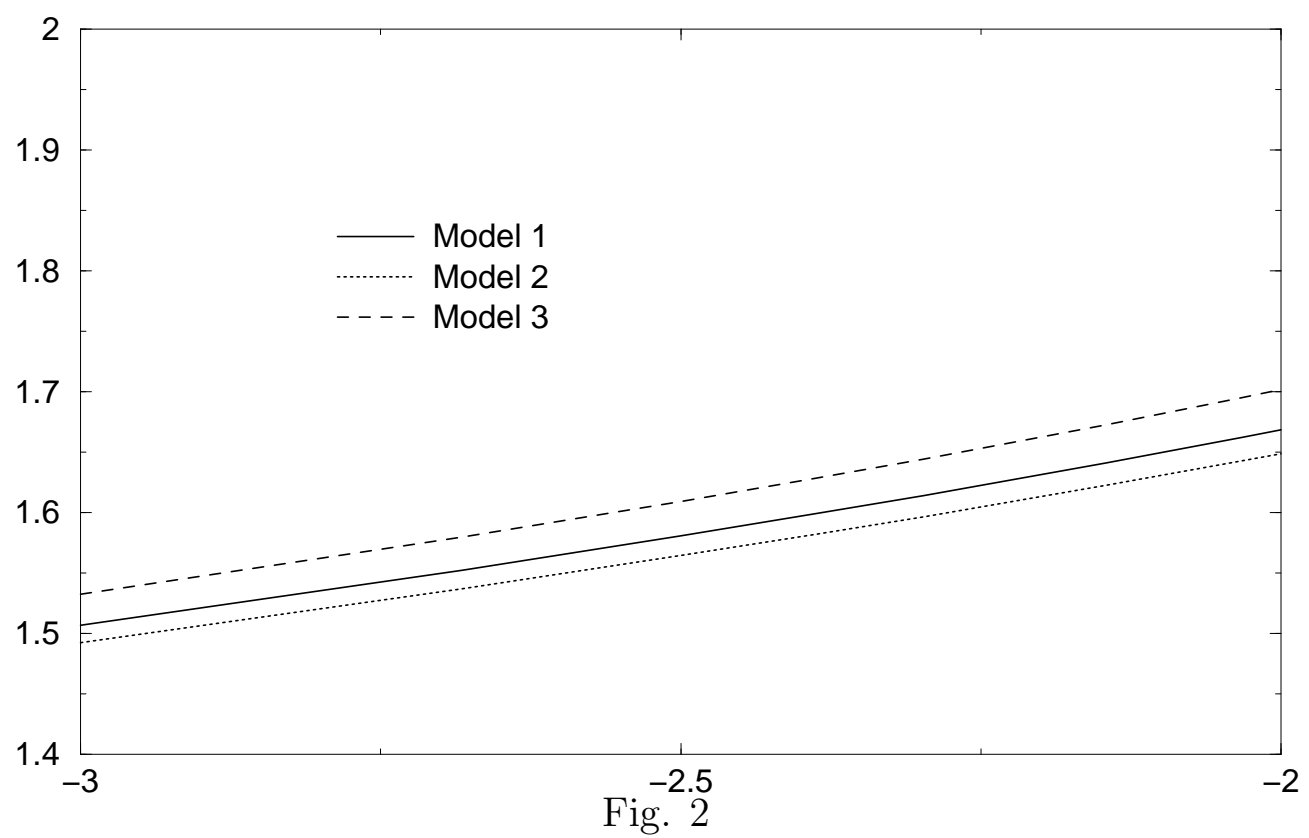




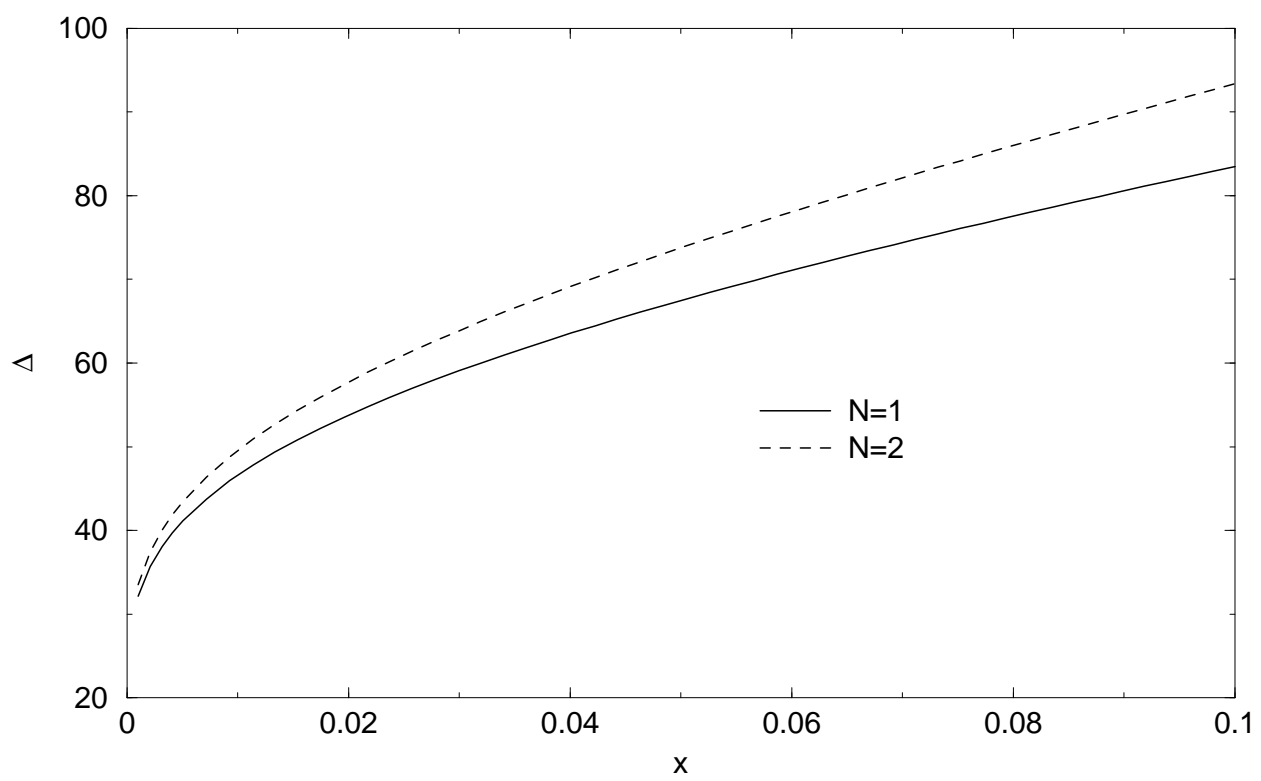

Fig. 3 


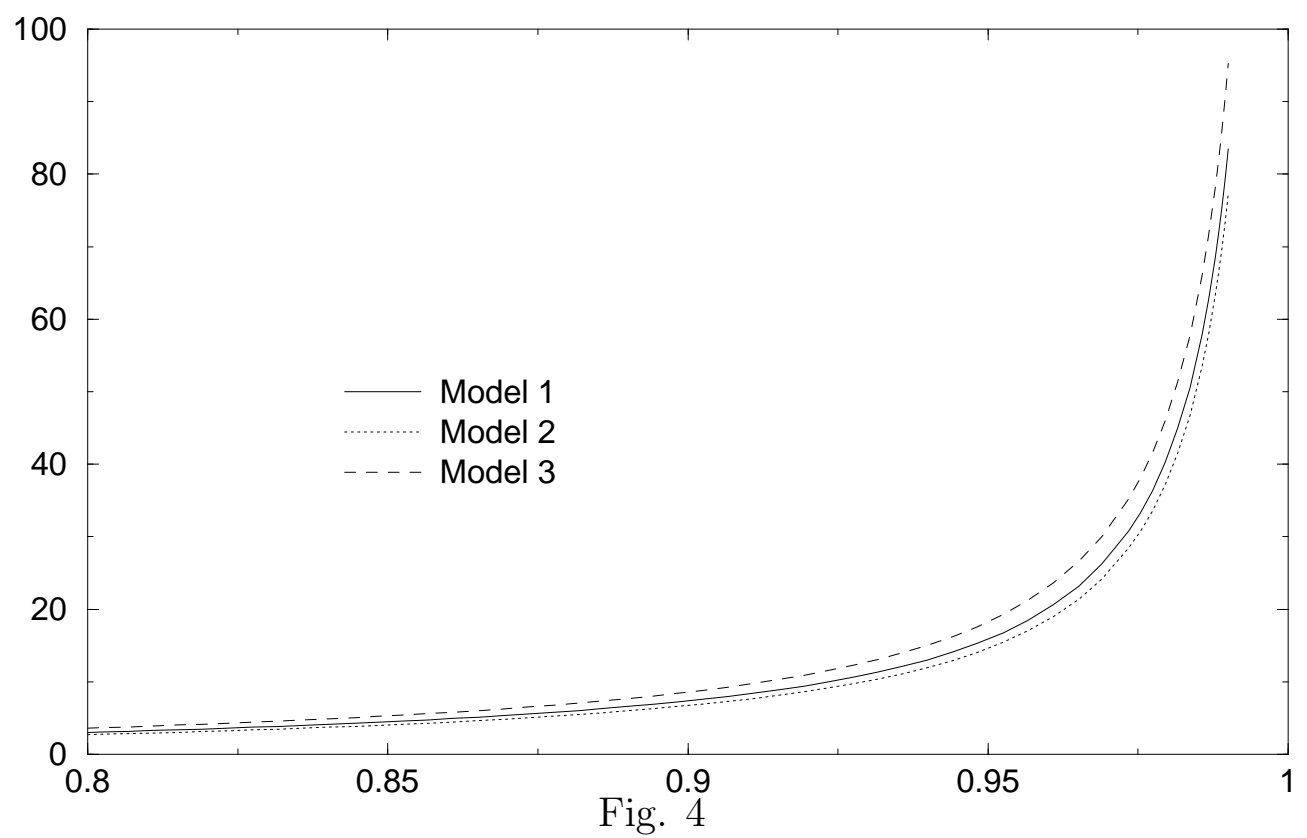

